\title{
Research Article \\ Effect of Pole Number on Generator End Winding Electromagnetic Force and Mechanical Response before and after RISC
}

\author{
Hong-Chun Jiang $\mathbb{D}$, Yu-Ling $\mathrm{He}(\mathbb{D}$, and Gui-Ji Tang \\ Department of Mechanical Engineering and Hebei Key Laboratory of Electric Machinery and Failure Prevention, North China \\ Electric Power University, Baoding 071003, China
}

Correspondence should be addressed to Hong-Chun Jiang; spring.jh@163.com

Received 4 October 2021; Accepted 23 November 2021; Published 23 December 2021

Academic Editor: Cheng Xu

Copyright (c) 2021 Hong-Chun Jiang et al. This is an open access article distributed under the Creative Commons Attribution License, which permits unrestricted use, distribution, and reproduction in any medium, provided the original work is properly cited.

\begin{abstract}
This paper comparatively studies on the end winding electromagnetic force and mechanical response for generators with different numbers of poles. The analytical expression of the end winding electromagnetic force is derived under the rotor winding interturn short circuit (RISC) considering the pole number. Meanwhile, the three-dimensional transient finite element simulation is carried on two generators with one-pair poles and three-pair poles. Then, the frequency composition and amplitude variation characteristics of the radial, axial, and tangential electromagnetic forces are analyzed. Further, the maximum stress and deformation on the end winding are calculated and the similarity and difference of the coil failure law are obtained for two kinds of generators. It is found that RISC will bring odd harmonics to electromagnetic force for one-pair pole generators but it will bring odd and fraction harmonics for multipair pole generators. Moreover, the max mechanical response under RISC will decrease for one-pair pole generators but it will increase for multipair pole generators.
\end{abstract}

\section{Introduction}

The generator is mainly composed of stator, rotor, and auxiliary components. When the rotor rotates, a changing magnetic field will be formed and the electromotive force and current will be excited on the stator winding. Further, the stator winding will generate alternating electromagnetic force under the action of field and current. The stator end winding is suspended outside the stator core, and alternating stress and vibration will be produced by electromagnetic force. Then, fatigue damage of the wire rod and insulation wear will be brought [1]. Due to the interturn insulation damage caused by the poor manufacturing process or copper wire structure defects, fatigue damage, or insulation aging caused by mechanical stress, the rotor interturn short circuit (RISC) occurs very easily. It is an electrical fault with high frequency in the actual working process of the generator. Such fault will not seriously affect the operation of the unit when they are slight in the early stage, and the generator can work with it.
But it will cause abnormal distribution of the air gap magnetic field [2], further affect the electromagnetic force and mechanical response of end winding, and aggravate the fatigue damage and vibration wear of some windings. Therefore, it is of great academic and practical significance to analyze the generator end winding electromagnetic force and mechanical response characteristics before and after RISC, so as to obtain the corresponding laws of winding fatigue failure and insulation wear. The study result can provide a basis for winding failure prevention and wear monitoring.

Reference [3] made an online detection of end leakage flux, and it is found that new frequency components will appear in the air gap magnetic motive force (MMF) when the generator is under RISC. Based on this principle, Yuguang et al. invented a new detection coil for the identification of interturn short circuit fault. When RISC happens, the detection coil port voltage appears even or fractional harmonics. However, when the stator interturn short circuit (SISC) happens, the port voltage only contains odd 


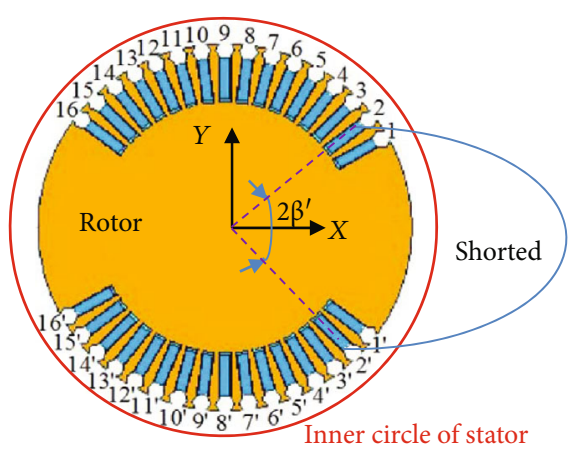

(a)

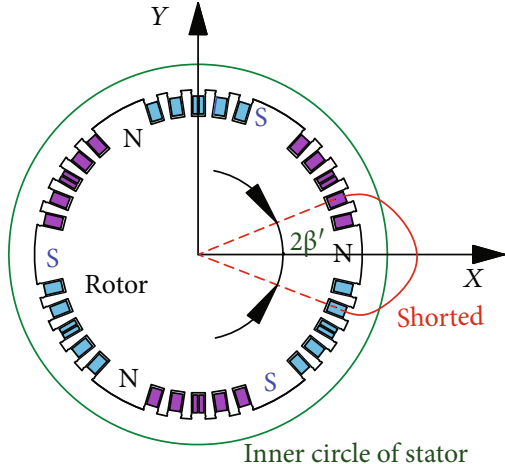

(b)

FiguRE 1: RISC: (a) QFSN-600-2YHG turbine generator $(p=1)$ and (b) MJF-30-6 prototype generator $(p=3)$.

harmonics [4]. The change of air gap MMF under RISC will cause the frequency change of magnetic pull force on the rotor surface, which is related to the stator current frequency and the pole number [5]. In addition, the difference between the actual generator electromagnetic power and the calculated virtual work value will increase with the occurrence of RICS [6]. Hongsen found that the average electromagnetic torque of the rotor will decrease when SISC happens [7]. Therefore, references [8-11] propose a composite diagnosis method based on stator and rotor vibration characteristics to determine the position and degree of RISC.

Moreover, the change of the air gap magnetic field will further affect the electromagnetic force of end winding. Reference [12] found that RISC will increase the amplitude of the odd harmonic component of stator winding electromagnetic force. This conclusion ignores the influence of the pole number on the reverse MMF, so its application is limited. Reference [13] took a generator with 3 pairs of poles as the object and proposed that the stress and deformation of some end windings decrease after RISC but the change is not obvious, so it does not have sufficient representation.

As an improvement, this paper mainly studies on the electromagnetic force and maximum mechanical response of the end winding before and after RISC and analyses the similarity and difference of generators with different numbers of poles. The remainder of this paper is constructed as follows. The qualitative equation of the end winding electromagnetic force under RISC is derived in Section 2. Then, the finite element analysis of the electromagnetic force and mechanical response are carried out in Section 3 and Section 4. Finally, the main conclusions are drawn up in Section 5.

\section{Theoretic Analysis}

2.1. Magnetic Flux Density (MFD) Analysis. In the normal condition, the MMF of the stator and rotor windings can be written as [14]

$$
\begin{gathered}
\left\{f_{s}(\alpha, t)=\sum_{n=1,3,5, \cdots,} F_{s n} \cos \left[n p\left(\omega_{r} t-\alpha\right)-\psi-0.5 \pi\right]\right. \\
=\sum_{n=1,3,5, \cdots,} F_{s n} \cos [n(\omega t-\alpha p)-\psi-0.5 \pi],
\end{gathered}
$$

$$
\left\{f_{r}(\alpha, t)=\sum_{n=1,3,5, \cdots,} F_{r n} \cos n p\left(\omega_{r} t-\alpha\right)=\sum_{n=1,3,5, \cdots,} F_{r n} \cos n(\omega t-\alpha p),\right.
$$

where $\alpha$ is the circumferential position, $p$ is the number of pole pair, $\psi$ is the internal power angle of the generator, $\omega_{r}$ is the angle velocity of the rotor, $\omega$ is the electricity frequency $\left(\omega=p \omega_{r}\right)$, and $F_{s n}$ and $F_{r n}$ are the $n^{\text {th }}$ harmonic amplitude of the stator and rotor winding MMF, respectively.

When the RISC happens, the shorted turns will produce a reverse MMF. The shorted position is defined as $\beta^{\prime}$ as shown in Figure 1, and $\beta^{\prime} \in(0 \sim \pi / p)$. According to the magnetic flux conservation law, the distribution of reverse MMF is shown in Figure 2 and it can be expressed as

$$
F_{d}(\alpha)=\left\{\begin{array}{l}
-\frac{I_{f} n_{m}\left(\pi-\beta^{\prime}\right)}{\pi}, \quad-\beta^{\prime} \leq \alpha \leq \beta^{\prime}, \\
\frac{I_{f} n_{m} \beta^{\prime}}{\pi}, \quad \text { others, }
\end{array}\right.
$$

where $I_{f}$ and $n_{m}$ are the current and number of shorted turns, respectively.

In the light of [13], the reverse MMF can be described as

$$
\begin{aligned}
F_{d}(\alpha) & =-\sum_{u=1}^{\infty} F_{d u} \cos u\left(\omega_{r} t-\alpha\right)=-\sum_{u=1}^{\infty} F_{d u} \cos \frac{u}{p}(\omega t-\alpha p), \\
F_{d u} & =\frac{2 I_{f} n_{m} \sin \left(u \beta^{\prime}\right)}{u \pi},
\end{aligned}
$$

where the absolution of $F_{d u}$ represents the $(u / p)^{\text {th }}$ harmonic amplitude of reverse MMF.

Because the rotor MMF is the main part, the influence of RISC on the stator MMF can be neglected. Therefore, the air gap MMF after RISC can be written as 


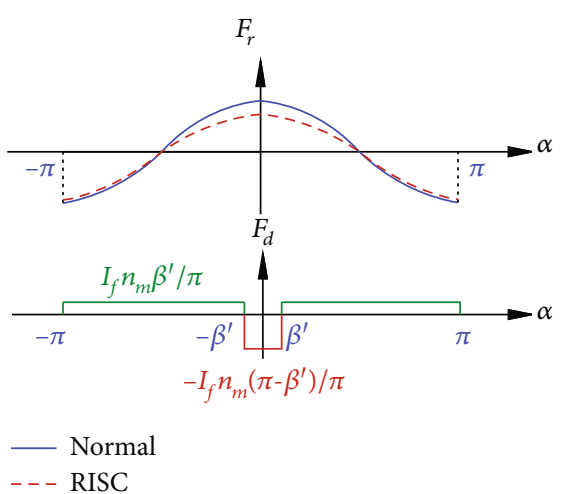

(a)

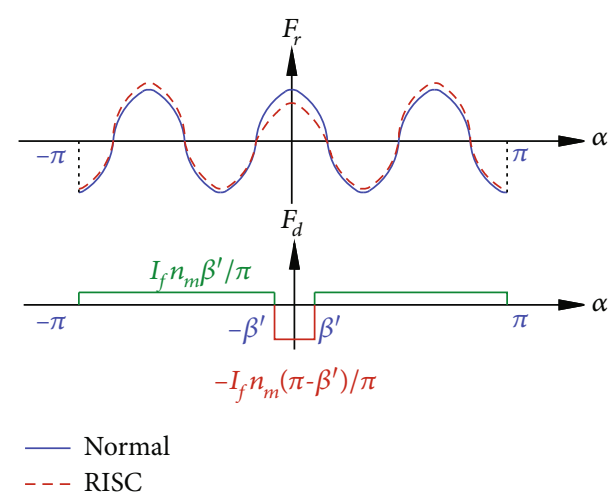

(b)

FIGURE 2: Inverse MMF (only the $1^{\text {st }}$ harmonic of the normal MMF is considered): (a) QFSN-600-2YHG turbine generator ( $p=1$ ) and (b) MJF-30-6 prototype generator $(p=3)$.

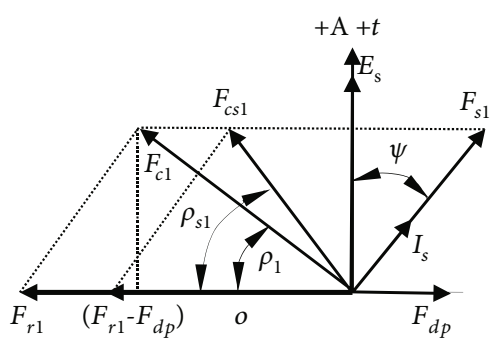

Figure 3: Diagram of fundamental MMF vectors $\left(F_{c 1}\right.$ and $F_{c s 1}$ represent the base harmonic of summed MMF in normal and RISC).

$$
\begin{gathered}
\left\{f_{s}(\alpha, t)=\sum_{n=1,3,5, \cdots,} F_{r n} \cos n p\left(\omega_{r} t-\alpha\right)+F_{s n} \cos \left[n p\left(\omega_{r} t-\alpha\right)-\psi-0.5 \pi\right]\right. \\
-\sum_{u=1}^{\infty} F_{d u} \cos u\left(\omega_{r} t-\alpha\right) \\
=\sum_{n=1,3,5, \cdots,} F_{c s n} \cos n\left(\omega t-\alpha p-\rho_{s n}\right)-\sum_{u=1,2,3, \cdots, u \neq n p} F_{d u} \cos \frac{u}{p}(\omega t-\alpha p) \\
\approx F_{c s 1} \cos \left(\omega t-\alpha p-\rho_{s 1}\right), \\
F_{c s 1}=\sqrt{F_{s 1}^{2} \cos ^{2} \psi+\left(F_{r 1}-F_{d p}-F_{s 1} \sin \psi\right)^{2}} \\
\rho_{s 1}=\arctan \frac{F_{s 1} \cos \psi}{F_{r 1}-F_{d p}-F_{s 1} \sin \psi}
\end{gathered}
$$

where $F_{c s n}$ is the $n^{\text {th }}$ harmonic amplitude of the summed MMF under RISC and $\rho_{s n}$ is the angle between the summed MMF and rotor MMF vector. The relationship of the fundamental harmonic MMF vectors is shown in Figure 3.

It can be indicated in equation (4) that the time component of rotating frequency is brought to the MMF under RISC and they are the $(u / p)^{\text {th }}$ harmonics. Moreover, RISC will result in the variation of the original odd harmonic amplitude and the changing tendency is decided by the pole number, shorted position, and order number (see equation (3)). Because $\beta^{\prime} \in(0 \sim \pi / p)$, sin $\left(p \beta^{\prime}\right)>0$ is constant, that is to say, $F_{d p}>0$. So, the fundamental harmonic amplitude of the summed MMF will be decreased and this conclusion is independent of the pole number.

Since RISC mainly affects the MMF and has little effect on the air gap magnetic permeance, the air-gap MFD at the iron core can be obtained by multiplying the RISC MMF and the normal permeance.

$$
\begin{aligned}
B_{s}(\alpha, t)= & f_{s}(\alpha, t) \Lambda_{0}=\Lambda_{0}\left[\sum_{n=1,3,5, \cdots,} F_{c s n} \cos n p\left(\omega_{r} t-\alpha-\frac{\rho_{s n}}{p}\right)\right. \\
& \left.-\sum_{u=1,2,3, \cdots, u \neq n p}^{\infty} F_{d u} \cos u\left(\omega_{r} t-\alpha\right)\right] \\
= & \Lambda_{0}\left[\sum_{n=1,3,5, \cdots,} F_{c s n} \cos n\left(\omega t-\alpha p-\rho_{s n}\right)-\sum_{u=1,2,3, \cdots, u \neq n p}^{\infty} F_{d u} \cos \frac{u}{p}(\omega t-\alpha p)\right] \\
\approx & \Lambda_{0} F_{c s 1} \cos \left(\omega t-\alpha p-\rho_{s 1}\right),
\end{aligned}
$$

where $\Lambda_{0}$ refers to the normal permeance $\left(\Lambda_{0}=\mu_{0} / \delta_{0}\right.$, where $\mu_{0}$ and $\delta_{0}$ are the vacuum permeability and normal air gap length, respectively).

As indicated in equation (5), it is evident that the MFD change tendency is consistent with MMF under RISC. The fundamental harmonic amplitude will decrease, and time components of rotating frequency will be brought. As shown in Table 1, the MFD mainly contains odd harmonic components such as $\omega, 3 \omega, 5 \omega$, and so on in normal condition. For a generator with one pair of poles, even harmonics will appear under RISC, such as $2 \omega, 4 \omega$, and $6 \omega$. For the generator with 3 pairs of poles, fractional and even harmonic components will be added, such as $\omega / 3,2 \omega / 3,4 \omega / 3,5 \omega / 3$, and $2 \omega$.

2.2. Electromagnetic Force Analysis. The main MFD can be achieved by the multiplication of rotor MMF and permeance.

$$
\begin{aligned}
B_{r}(\alpha, t)= & \Lambda_{0}\left[\sum_{n=1,3,5, \cdots,}\left(F_{r n}-F_{d(n p)}\right) \cos n p\left(\omega_{r} t-\alpha\right)\right. \\
& \left.-\sum_{u=1,2,3, \cdots, u \neq n p} F_{d u} \cos u\left(\omega_{r} t-\alpha\right)\right] .
\end{aligned}
$$


TABLE 1: Component comparison of MMF and electromagnetic force.

\begin{tabular}{|c|c|c|c|c|}
\hline & \multicolumn{2}{|c|}{$p=1\left(\omega=\omega_{r}\right)$} & \multicolumn{2}{|c|}{$p=3\left(\omega=3 \omega_{r}\right)$} \\
\hline & $M M F(\mathrm{MFD})$ & Electromagnetic force & $M M F(\mathrm{MFD})$ & Electromagnetic force \\
\hline \multirow{3}{*}{ Component in normal } & $\omega_{r}(\omega)$ & $2 \omega_{r}(2 \omega)$ & $3 \omega_{r}(\omega)$ & $6 \omega_{r}(2 \omega)$ \\
\hline & $3 \omega_{r}(3 \omega)$ & $4 \omega_{r}(4 \omega)$ & $9 \omega_{r}(3 \omega)$ & $12 \omega_{r}(4 \omega)$ \\
\hline & $5 \omega_{r}(5 \omega)$ & $6 \omega_{r}(6 \omega)$ & $15 \omega_{r}(5 \omega)$ & $18 \omega_{r}(6 \omega)$ \\
\hline \multirow{5}{*}{ New component under RISC } & & & $\omega_{r}(\omega / 3)$ & $\omega_{r}(\omega / 3)$ \\
\hline & $2 \omega_{r}(2 \omega)$ & $\omega_{r}(\omega)$ & $2 \omega_{r}(2 \omega / 3)$ & $2 \omega_{r}(2 \omega / 3)$ \\
\hline & $4 \omega_{r}(4 \omega)$ & $3 \omega_{r}(3 \omega)$ & $4 \omega_{r}(4 \omega / 3)$ & $3 \omega_{r}(\omega)$ \\
\hline & $6 \omega_{r}(6 \omega)$ & $5 \omega_{r}(5 \omega)$ & $5 \omega_{r}(5 \omega / 3)$ & $4 \omega_{r}(4 \omega / 3)$ \\
\hline & & $\ldots$ & $6 \omega_{r}(2 \omega)$ & $5 \omega_{r}(5 \omega / 3)$ \\
\hline
\end{tabular}

According the electromagnetic induction rule, the stator current can be described as

$$
\left\{\begin{array}{l}
I_{s}(t)=\frac{2 p q w_{s} k_{w}}{a} L v\left(\sum_{n=1,3,5 \cdots}\left(F_{r n}-F_{d(n p)}\right) \cos n p\left(\omega_{r} t-\alpha\right)-\sum_{u=1,2,3 \cdots \text { and } u \neq n p} F_{d u} \cos u\left(\omega_{r} t-\alpha\right)\right) / Z \\
=\sum_{n=1,3,5 \cdots} I_{s n} \cos \left[n p\left(\omega_{r} t-\alpha\right)-\psi-\pi / 2\right]+\sum_{u=1,2,3 \cdots \text { and } u \neq n p} I_{s u} \cos \left[u\left(\omega_{r} t-\alpha\right)-\psi-\pi / 2\right] \\
\left.\left.=\sum_{n=1,3,5 \cdots} I_{s n} \cos [n(\omega t-\alpha p)-\psi-\pi / 2)\right]+\sum_{u=1,2,3 \cdots \text { and } u \neq n p} I_{s u} \cos \left[\frac{u}{p}(\omega t-\alpha p)-\psi-\pi / 2\right)\right] \\
I_{s n}=\frac{2 p q}{a|Z|} w_{s} k_{w n} \Lambda_{0} L v\left(F_{r n}-F_{d(n p)}\right) \quad n=1,3,5 \cdots \\
I_{s u}=\frac{2 p q}{a|Z|} w_{s} k_{w u} \Lambda_{0} L v F_{d u} \quad u=1,2,3 \cdots \text { and } u \neq n p
\end{array}\right.
$$

where $I_{s n}$ is the $n^{\text {th }}$ harmonic amplitude of stator current and $I_{s u}$ is $(u / p)^{\text {th }}$ harmonic amplitude. $L$ is the effective axial length of the winding, $v$ is the velocity that the coil bar cuts the magnetic flux lines, $Z$ is the impedance of the winding, $q$ is the slot number per phase, $w_{s}$ is the turn number per coil, and $k_{w n}$ is the winding factor of the $n$th harmonic (for details, see [14]).

Through the ampere force law, the electromagnetic force of end coil point $K$ can be expressed as

$$
\left\{\begin{array}{l}
\overrightarrow{F_{I k s}}=f_{k} \overrightarrow{B_{s}} \times \overrightarrow{I_{s}} d l=\left\{\overrightarrow{F_{I k x s}}, \overrightarrow{F_{I k v s}}, \overrightarrow{F_{I k z s}}\right\} d l \\
F_{I k s}\left(\alpha_{I}+\alpha_{k}, t\right)=f_{k} B_{s} I_{s} \sin \theta_{k} d l \\
\approx \frac{p q}{a|Z|} f_{k} q w_{s} L v \Lambda_{0}^{2} \sin \theta_{k} d l \\
\left(\begin{array}{c}
\sum_{n=1,3,5 \cdots j=1,3,5 \cdots} k_{w n}\left(F_{r n}-F_{d(n p)}\right) F_{c s j}\left[\begin{array}{c}
\cos p\left[(n+j)\left(\omega_{r} t-\alpha_{I}\right)-j \alpha_{k}-j \rho_{s n} / p-\psi-\pi / 2\right] \\
+\cos p\left[(n-j)\left(\omega_{r} t-\alpha_{I}\right)-j \alpha_{k}-j \rho_{s n} l p+\psi+\pi / 2\right]
\end{array}\right] \\
-\sum_{n=1,3,5 \cdots j=1,2,3 \cdots ; j \neq n p} k_{w n}\left(F_{r n}-F_{d(n p)}\right) F_{d j}\left[\begin{array}{c}
\cos \left[(n p+j)\left(\omega_{r} t-\alpha_{I}\right)-j \alpha_{k}-\psi-\pi / 2\right] \\
+\cos \left[(n p-j)\left(\omega_{r} t-\alpha_{I}\right)-j \alpha_{k}+\psi+\pi / 2\right]
\end{array}\right] \\
-\sum_{n=1,3,5 \cdots j=1,2,3 \cdots ; j \neq n p} k_{w j} F_{d j} F_{c s n}\left[\begin{array}{c}
\cos \left[(n p+j)\left(\omega_{r} t-\alpha_{I}\right)-n p \alpha_{k}-n \rho_{s n}-\psi-\pi / 2\right] \\
+\cos \left[(n p-j)\left(\omega_{r} t-\alpha_{I}\right)-n p \alpha_{k}-n \rho_{s n}+\psi+\pi / 2\right]
\end{array}\right]
\end{array}\right. \\
\approx \frac{p q}{a|Z|} f_{k} q w_{s} k_{w 1} L v \Lambda_{0}^{2} \sin \theta_{k} d l\left(F_{r 1}-F_{d p}\right) F_{c s 1}\left[\begin{array}{c}
\cos p\left[2\left(\omega_{r} t-\alpha_{I}\right)-\alpha_{k}-\rho_{s n} / p-\psi-\pi / 2\right] \\
+\cos p\left[\alpha_{k}+\rho_{s n} / p-\psi-\pi / 2\right]
\end{array}\right]
\end{array}\right.
$$




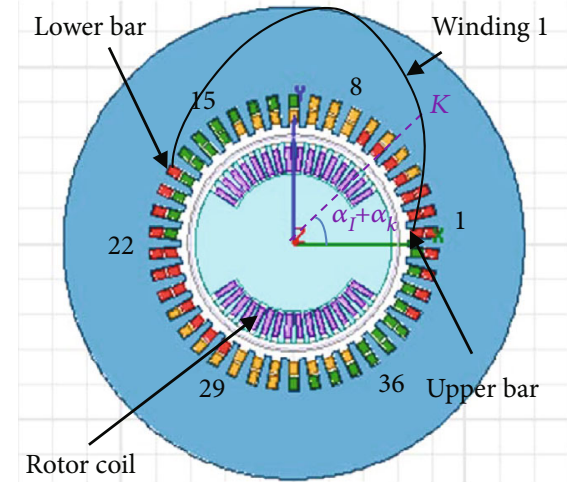

(a)

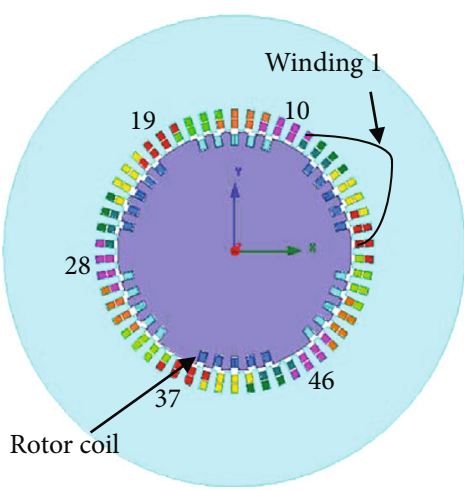

(b)

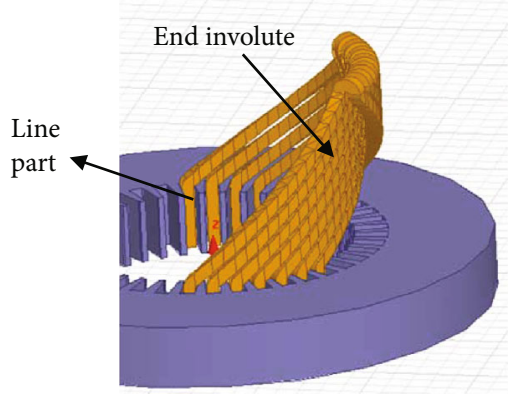

(c)

Figure 4: Layout of wingdings: (a) QFSN-600-2YHG generator, (b) MJF-30-6 simulator, and (c) 3D model of stator and windings.

where $F_{I k x s}, F_{I k y s}$, and $F_{I k z s}$ are rectangular coordinate components of electromagnetic force under RISC. $\alpha_{I}$ is the circumferential angle of the upper layer line part in the $I^{\text {th }}$ stator slot, and $\alpha_{I}+\alpha_{k}$ refers to the position of the point $K$ as shown in Figure 4(a). $\theta_{k}$ is the angle between the magnetic flux density and the stator current at point $K$, and $f_{k}$ is the MFD modification coefficient.

Through coordinate transformation and integral calculation, the radial, tangential, axial electromagnetic force components, and resultant force of the end winding under composite fault can be obtained by

$$
\begin{array}{r}
F_{I r s}=\int_{l_{\text {end }}}\left(F_{I k x s} \cos \theta+F_{I k y s} \sin \theta\right) d l, \\
F_{I t s}=\int_{l_{\text {end }}}\left(-F_{I k x s} \sin \theta+F_{I k y s} \cos \theta\right) d l, \\
F_{I a s}=\int_{l_{\text {end }}} F_{I k z s} d l,
\end{array}
$$$$
F_{I s}=\sqrt{\left(\int_{l_{\text {end }}} F_{I k x s} d l\right)^{2}+\left(\int_{l_{\text {end }}} F_{I k y s} d l\right)^{2}+\left(\int_{l_{\text {end }}} F_{I k z s} d l\right)^{2}},
$$

where $\theta$ is the vector angle of the cylinder coordinate of point $K$ and $l_{\text {end }}$ is the coil curve of the end winding.
According to equation (8), in addition to $2 n p \omega_{r}(n=1$, $2,3, \cdots$, components, the electromagnetic force contains other time components of rotating frequency after RISC. As shown in Table 1, the electromagnetic force in normal condition mainly contains even harmonics such as $2 \omega, 4 \omega$, and $6 \omega$. When RISC occurs, odd harmonics will appear for the generator with one pair of poles, such as $\omega, 3 \omega$, and $5 \omega$. But fractional and odd harmonic components will be added for the generator with 3 pairs of poles, such as $\omega / 3,2 \omega / 3, \omega, 4 \omega / 3$, and $5 \omega / 3$.

On the other hand, in RISC condition, the original even harmonic amplitude of $2 n p \omega_{r}(n=1,2,3, \cdots$,$) will$ change and the tendency is related with the reverse MMF. Because $F_{d p}>0$ exists for the fundamental harmonic of reverse MMF and the summed MMF will decrease (see equation (4) and Figure 3, $F_{c s 1}<F_{c 1}$ ), therefore the $2 p \omega_{r}$ component (that is, the second harmonic) amplitude will decrease. This conclusion has no relationship with the pole number.

\section{Study Object and Simulation Settings}

In this paper, the electromagnetic force and mechanical response of end winding in the QFSN-600-2YHG turbogenerator and MJF-30-6 fault simulator are simulated by the finite element method. The two objects have one pair of poles and three pairs of poles respectively. The main parameters can be found in references $[13,14]$, and the layout of windings is shown in Figures 4(a) and 4(b). 


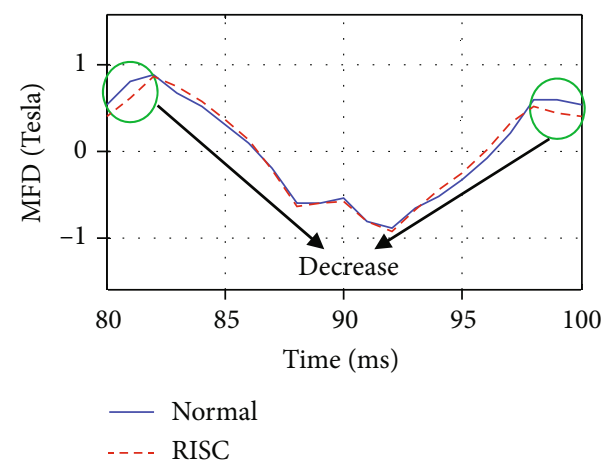

(a)

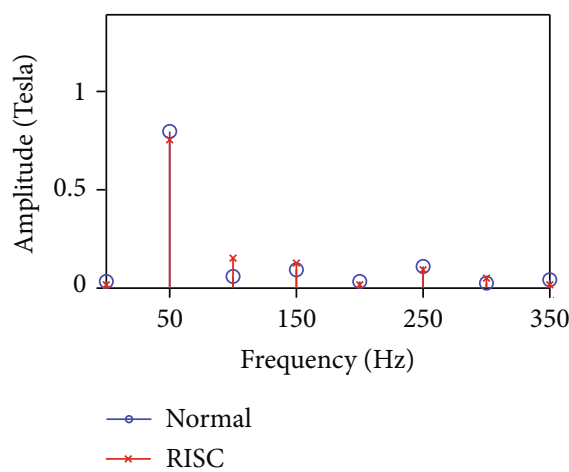

(b)

FIGURE 5: Radial flux density of the QFSN-600-2YHG generator: (a) time wave and (b) spectrum.

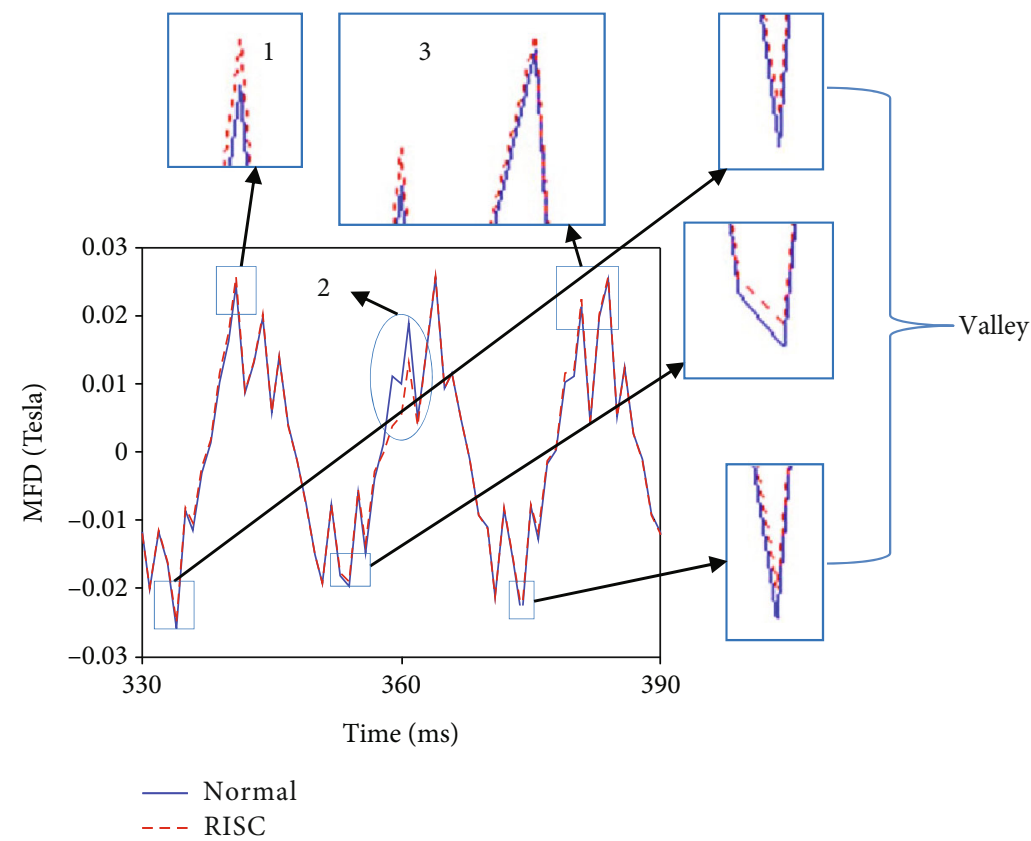

(a)

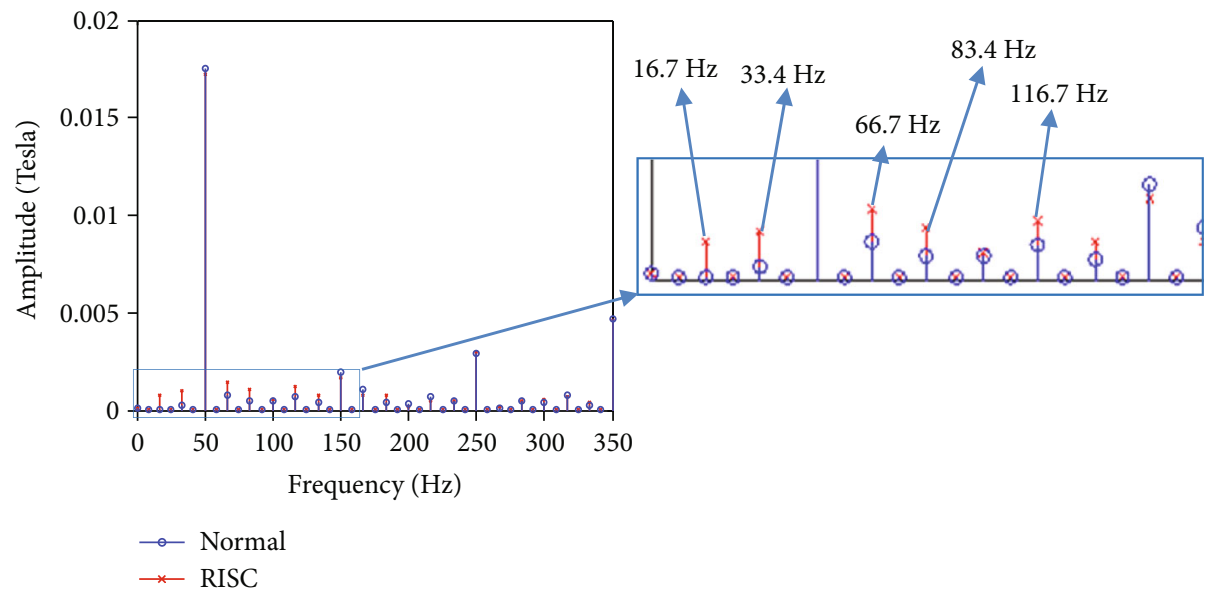

(b)

Figure 6: Radial flux density of the MJF-30-6 simulator: (a) time wave and (b) spectrum. 


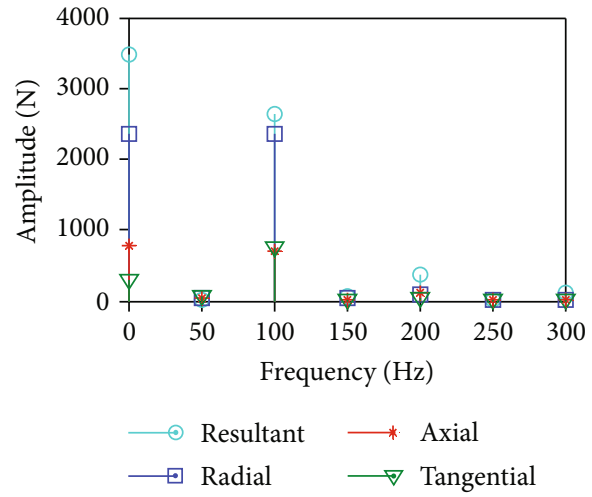

(a)

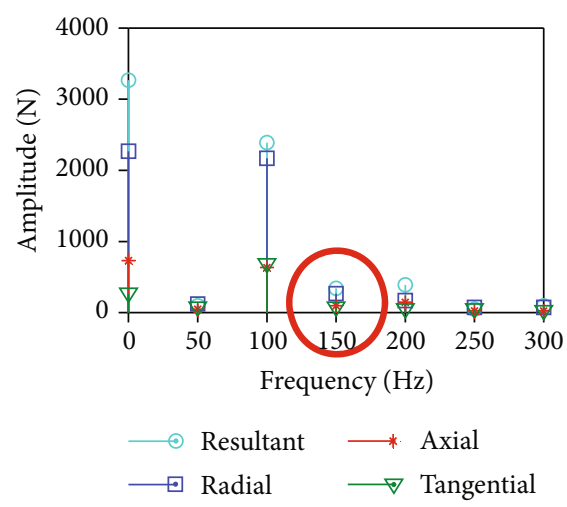

(b)

FIgURE 7: Electromagnetic force spectrum of the QFSN-600-2YHG generator: (a) in normal and (b) under RISC.

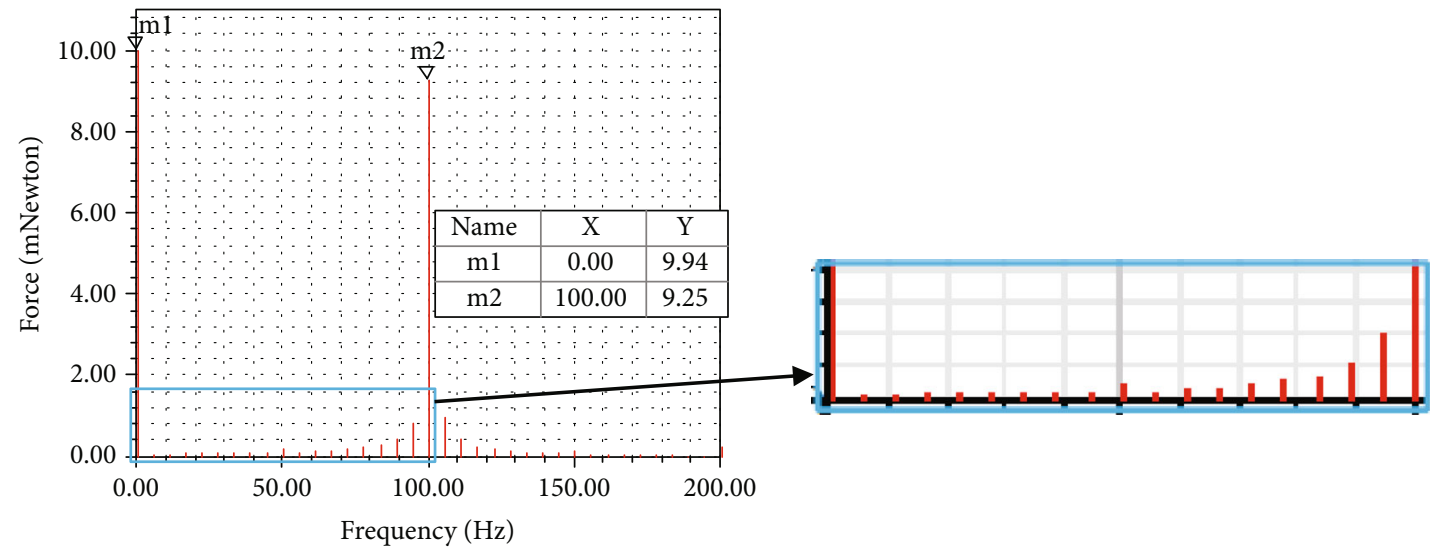

(a)

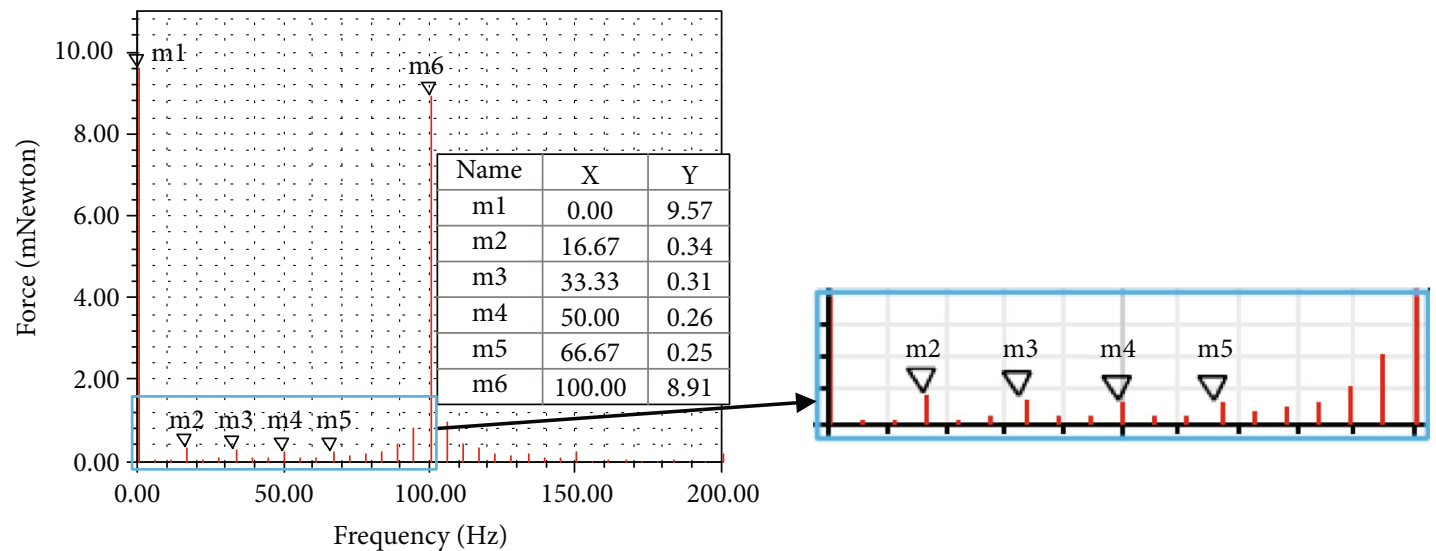

(b)

FIGURE 8: Electromagnetic force spectrum of the MJF-30-6 simulator: (a) in normal and (b) under RISC.

During the simulation, for the QFSN-600-2YHG turbogenerator, the rotor excitation current is set as a rated value of $4128 \mathrm{~A}$, the rotor speed is set as a synchronous speed of $3000 \mathrm{rpm}$, the step length is $0.0005 \mathrm{~s}$, and the simulation time is set as $0.12 \mathrm{~s}$ (that is, 6 cycles for the rotor). For the MJF-30-6 fault simulator, the rotor excitation current is set to the rated value of $1.8 \mathrm{~A}$, the rotor speed is set to $1000 \mathrm{rpm}$, the step size is set to $0.001 \mathrm{~s}$, and the simulation calculation time is $0.36 \mathrm{~s}$ (that is, also 6 cycles for the rotor).

\section{Simulation Result}

\subsection{MFD Result}

4.1.1. QFSN-600-2YHG Turbogenerator $\left(p=1, \omega=\omega_{r}=50\right.$ $\mathrm{Hz}$ ). The radial MFD wave and spectrum at $0^{\circ}$ before and after RISC are shown in Figure 5. It can be seen in Figure 5(a) that the MFD matches approximately with cosine. After RISC, it decreases significantly at the peak 


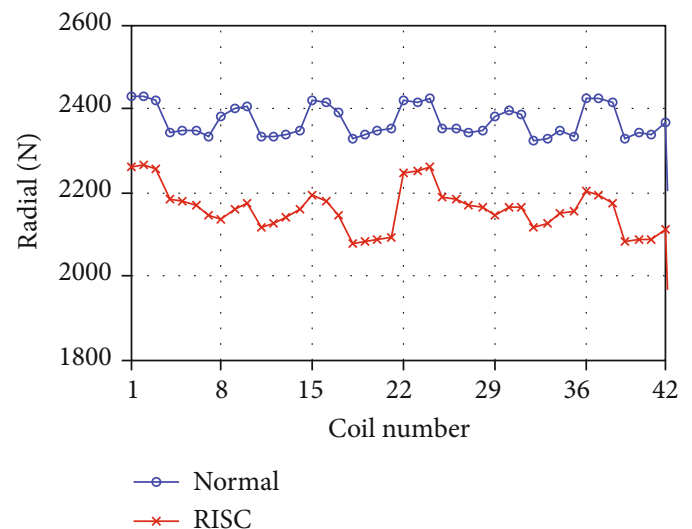

(a)
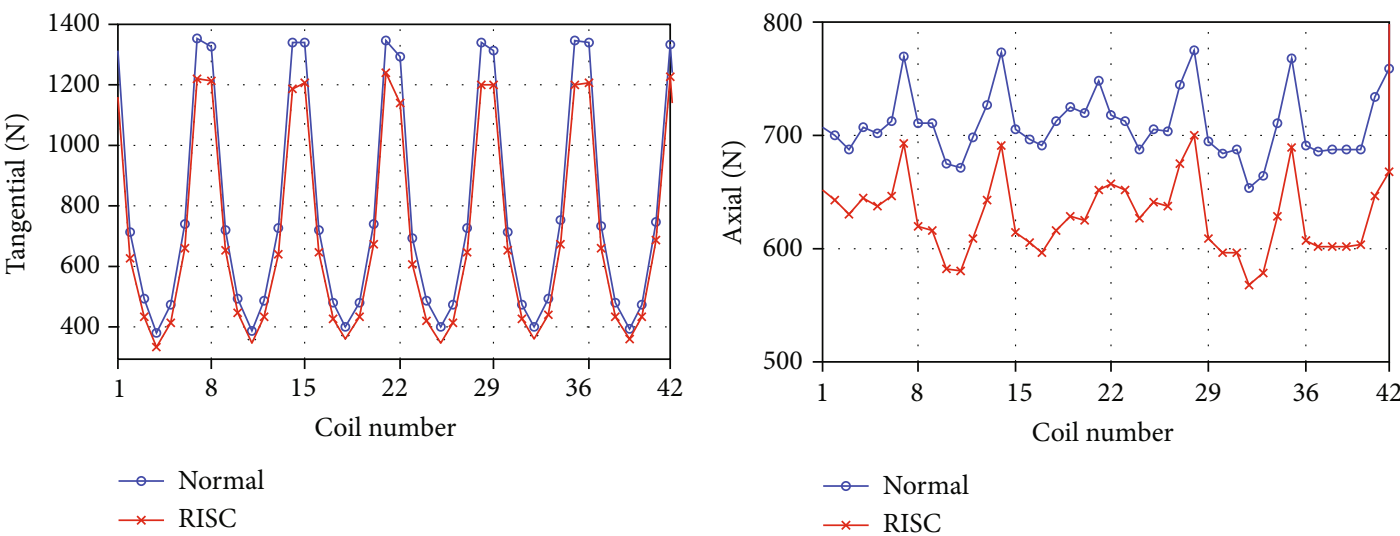

(b)

(c)

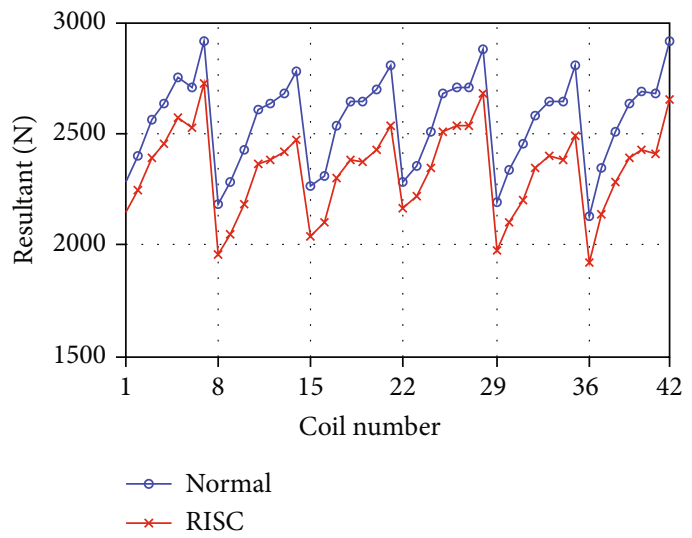

(d)

FIGURE 9: Second harmonic amplitude of electromagnetic force: (a) radial direction, (b) tangential direction, (c) axial direction, and (d) resultant force.

and the change at the valley is not obvious. This result is similar to the MMF (see Figure 2(a)).

Moreover, it indicates in Figure 5(b) that the MFD includes the obvious $50 \mathrm{~Hz}$ (fundamental harmonic $\omega$ ), $150 \mathrm{~Hz}$, and $250 \mathrm{~Hz}$ (that is, $3 \omega$ and $5 \omega$ ) components. Meantime, the amplitude increases at $100 \mathrm{~Hz}$ (that is, 2 $\omega)$ after RISC and it decreases at $50 \mathrm{~Hz}$. This conclusion is consistent with the theoretical analysis result of equation (5).
4.1.2. MJF-30-6 Fault Simulator $\left(p=3, \omega_{r}=16.7 \mathrm{~Hz}, \omega=50\right.$ $\mathrm{Hz}$ ). The radial MFD wave and spectrum at $0^{\circ}$ are shown in Figure 6. It shows in Figure 6(a) that, after RISC, the MFD decreases obviously at the no. 2 peak, increases slightly at the no. 1 and no. 3 peaks, and decreases slightly at the three valleys. So, the variation law is similar to MMF in Figure 2(b).

Further, it can be seen in Figure 6(b) that the magnetic density includes the obvious $50 \mathrm{~Hz}$ (fundamental harmonic $\omega), 150 \mathrm{~Hz}$, and $250 \mathrm{~Hz}$ (that is, $3 \omega$ and $5 \omega$ ) components. 


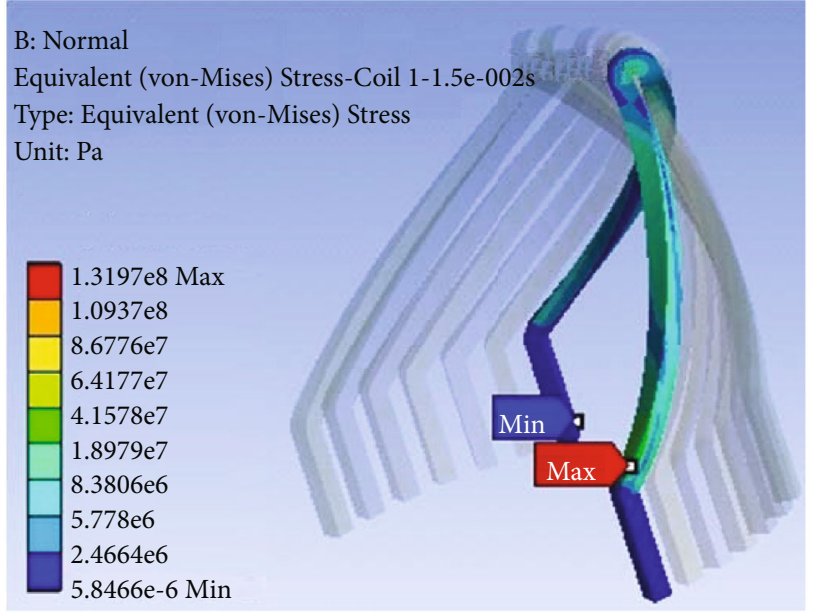

(a)

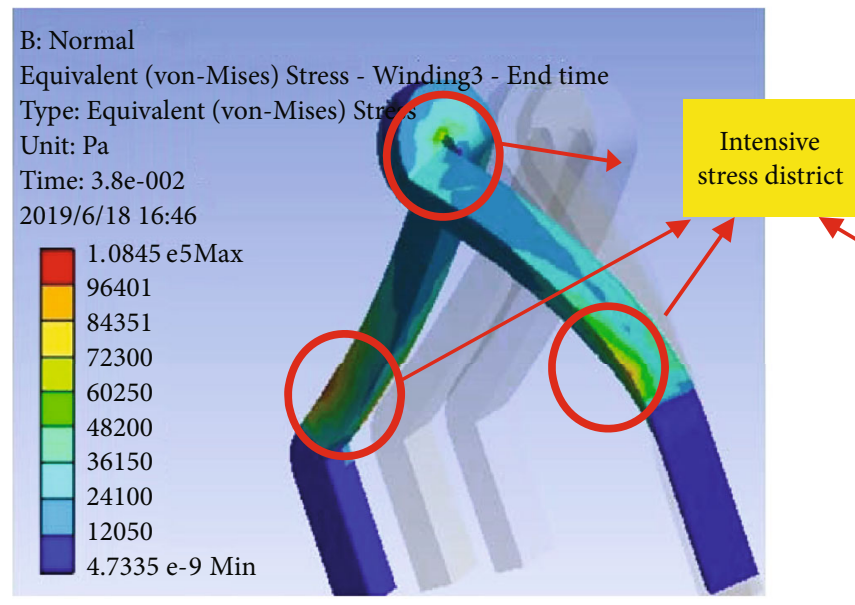

(c)

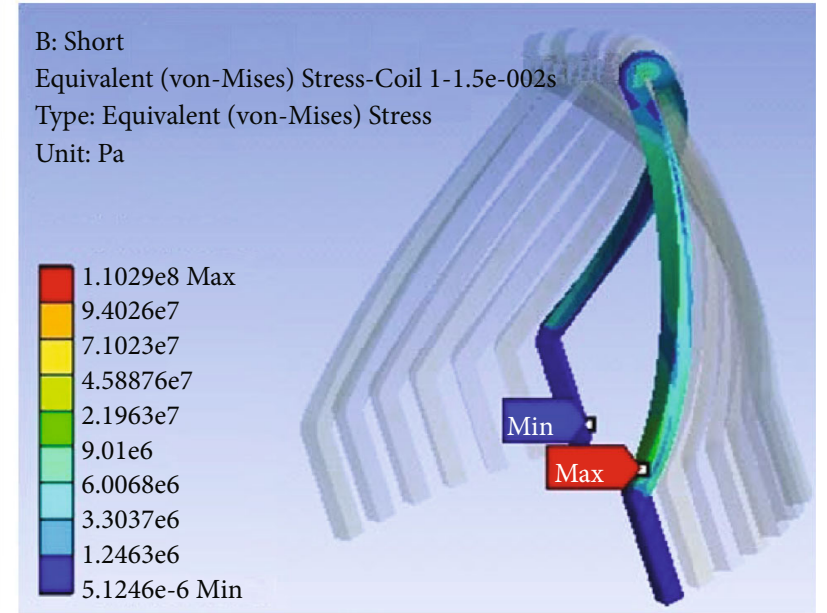

(b)

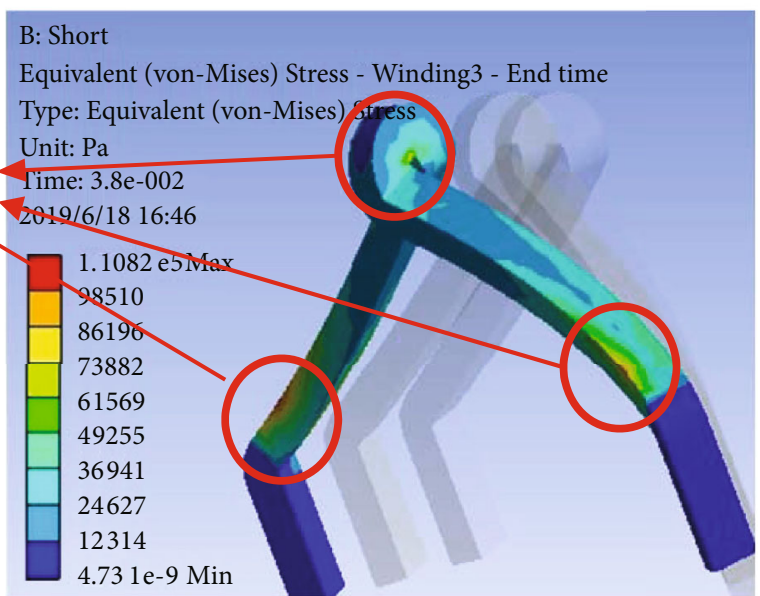

(d)

FIgURE 10: Stress distribution before and after RISC: (a, b) QFSN-600-2YHG generator in normal and RISC, respectively; (c, d) MJF-30-6 simulator in normal and RISC, respectively.

At the same time, the amplitude at $50 \mathrm{~Hz}$ decreases after RISC and it increase at $16.7 \mathrm{~Hz}, 33.4 \mathrm{~Hz}, 66.7 \mathrm{~Hz}$ (that is, $\omega$ $13,2 \omega / 3$, and $4 \omega / 3$ ), and so on. These results reflect the correction of theoretic equation (5).

4.2. Electromagnetic Force Result. The frequency components of electromagnetic force are shown in Figure $7(p=1)$ and Figure $8(p=3)$. Here, $p$ is the number of pole pairs.

4.2.1. QFSN-600-2YHG Turbogenerator $\left(p=1, \omega=\omega_{r}=50\right.$ $\mathrm{Hz}$ ). The electromagnetic force of each end coil can be obtained through finite element analysis. Taking phase A coils as an example, the electromagnetic force of coil 7 is the largest and its force spectrum before and after RISC is shown in Figure 7. It can be seen from the comparison that, in addition to the obvious DC constant, harmonics at $100 \mathrm{~Hz}$ and $200 \mathrm{~Hz}$ (that is, $2 \omega$ and $4 \omega$ ), there is a weak harmonic at $150 \mathrm{~Hz}(3 \omega)$ after RISC. This is consistent with the conclusion in theoretical analysis equation (8).

Because the second harmonics is the most obvious, its amplitude for 42 end coils is shown in Figure 9. It is shown that the force amplitude of coils $60^{\circ}$ apart is still approximately equal and the amplitude of interphase coils is the largest. However, the second harmonic amplitude of electromagnetic force decreases after RISC. Accordingly, the vibration wear of the coil at second harmonic will also be reduced.

4.2.2. MJF-30-6 Fault Simulator $\left(p=3, \omega_{r}=16.7 \mathrm{~Hz}, \omega=50\right.$ $\mathrm{Hz}$ ). The electromagnetic force of coil 2 is the largest in the phase A coils, and its force spectrum before and after RISC is shown in Figure 8. It indicates that there are obvious DC constants and $100 \mathrm{~Hz}$ (that is, second harmonic) components. After RISC, weak components at $16.7 \mathrm{~Hz}, 33.3 \mathrm{~Hz}$, and $50 \mathrm{~Hz}$ (that is, $\omega / 3,2 \omega / 3$, and $\omega$ ) increase and the harmonic amplitude at $100 \mathrm{~Hz}$ decreases from $9.25 \mathrm{mN}$ to $8.91 \mathrm{mN}$. It well verified the theoretic equation (8).

In summary, the second harmonic amplitude of electromagnetic force decreases after RISC, as shown in Figure 9 $(p=1)$ and Figure $8(p=3)$. And this conclusion has no relationship with the pole number. 


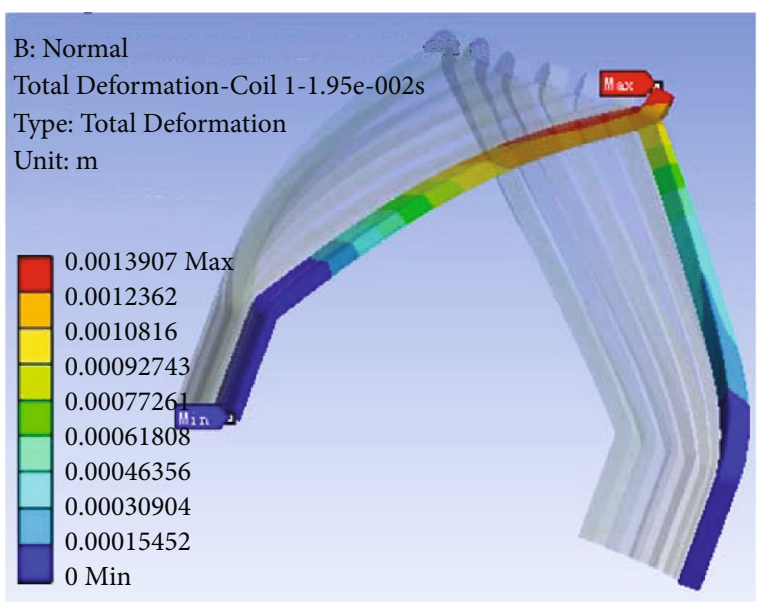

(a)

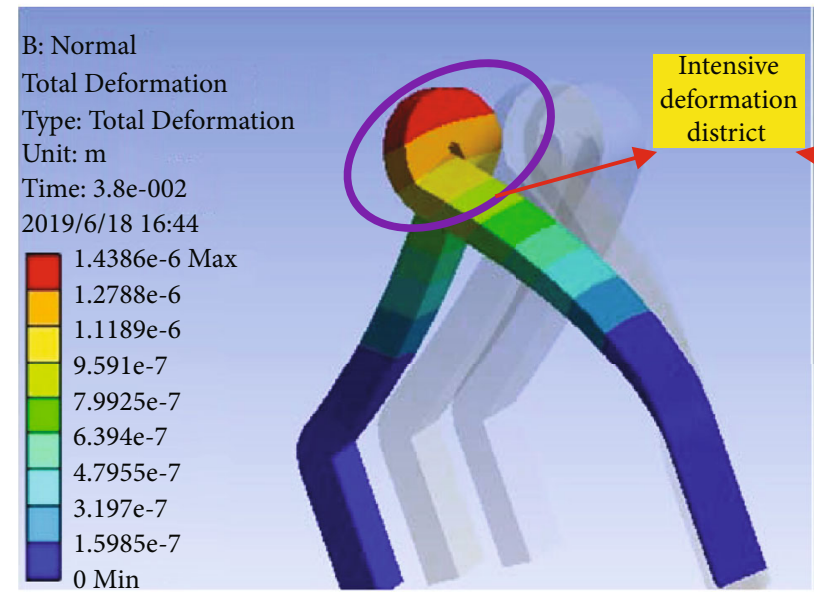

(c)

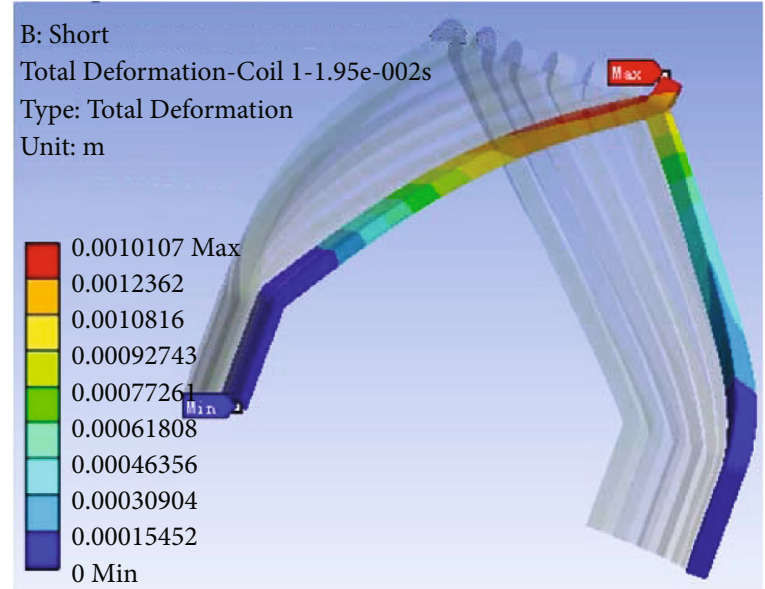

(b)

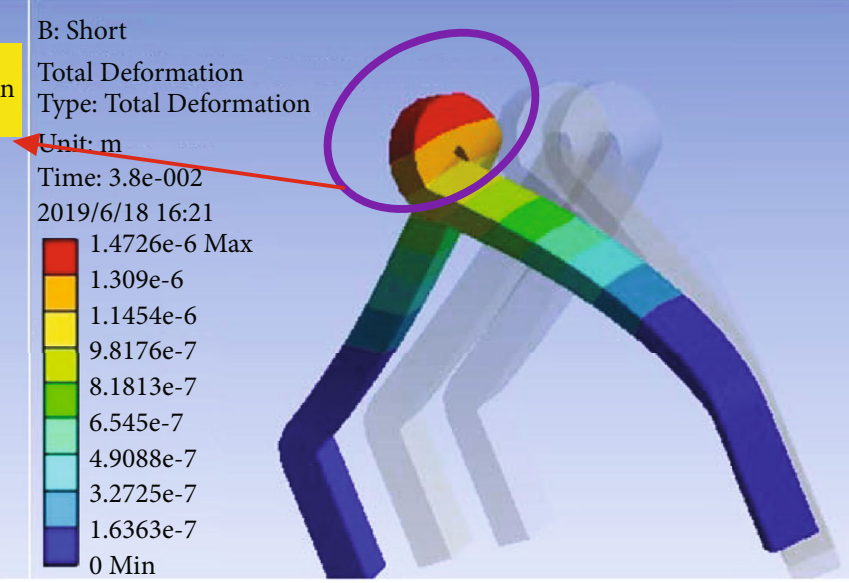

(d)

FIGURE 11: Deformation distribution before and after RISC: (a, b) QFSN-600-2YHG generator in normal and RISC, respectively; (c, d) MJF30-6 simulator in normal and RISC, respectively.

4.3. Mechanical Response Result. Through the finite element calculation of the mechanical structure, the maximum stress and deformation of each coil of phase A are obtained. The response of the no. 1 coil is the largest for the QFSN-6002YHG turbogenerator, and the no. 3 coil is the largest for the MJF-30-6 fault simulator. Therefore, these two coils are selected as representation to analyze the response characteristics.

4.3.1. Equivalent Stress. The maximum stress distribution of end winding for the QFSN-600-2YHG turbogenerator and MJF-30-6 fault simulator is shown in Figure 10. It indicates that the max stress occurs on the root part and the nose part also has a larger stress. Moreover, the max stress is reduced from $132 \mathrm{MPa}$ to $110 \mathrm{MPa}$ after RISC for the QFSN-6002YHG turbogenerator (see Figures 10 (a) and 10(b)) but it is increased from $1.08 \times 10^{5} \mathrm{~Pa}$ to $1.11 \times 10^{5} \mathrm{~Pa}$ for the MJF-30-6 fault simulator (see Figures $10(\mathrm{c})$ and 10(d)). This result is related to the change of MFD. The max value of MFD decreases for the QFSN-600-2YHG turbogenerator and increases for the MJF-30-6 fault simulator as shown in Figures 5(a) and 6(a).
4.3.2. Total Deformation. The maximum deformation distribution of end winding for the QFSN-600-2YHG turbogenerator and MJF-30-6 fault simulator is exhibited in Figure 11. It shows that the max deformation on the nose part is the largest. In the meantime, the max deformation decreases from $1.39 \mathrm{~mm}$ to $1.01 \mathrm{~mm}$ after RISC for the QFSN-6002YHG turbogenerator (see Figures 11(a) and 11(b)) but it increases from $1.44 \times 10^{-6} \mathrm{~m}$ to $1.47 \times 10^{-6} \mathrm{~m}$ for the MJF30-6 fault simulator (see Figures $11(\mathrm{c})$ and $11(\mathrm{~d})$ ). And the reason is the same as the above stress change.

\section{Conclusion}

In this paper, the electromagnetic force and mechanical response of stator end winding are comparatively analyzed for two generators with one pair of poles and 3 pairs of poles, respectively. It is found that RISC can decrease the second harmonic (that is, $100 \mathrm{~Hz}$ ) amplitude of the electromagnetic force and this conclusion has no relationship with the pole number. In the meantime, odd harmonics (that is, $50 \mathrm{~Hz}, 150 \mathrm{~Hz}$, and so on) will be brought to electromagnetic force after RISC for one-pair pole generators 
but the new components are odd and fraction harmonics (16.7 Hz, 33.3 Hz, and $50 \mathrm{~Hz}$, for example) for 3-pair pole generators. Moreover, the $\max$ stress and deformation under RISC will decrease for one-pair pole generators and increase for 3-pair pole generators. So the RISC is more dangerous for multipair generator winding damage. The conclusions can lay a foundation for the reverse suppression of end winding fatigue failure and insulation wear.

\section{Data Availability}

The data used to support the findings of this study are available from the corresponding author upon request.

\section{Conflicts of Interest}

All of the authors declare that there is no interest conflict regarding the publication of this paper.

\section{Acknowledgments}

This work is supported by the National Natural Science Foundation of China (51777074) and the Fundamental Research Funds for Central Universities (2017MS146).

\section{References}

[1] J. A. Tegopoulos, "Forces on the end winding of turbinegenerators II - determination of forces," IEEE Transactions on Power Apparatus and Systems, vol. 85, no. 2, pp. 114-122, 1966.

[2] Y. L. He, M. Q. Ke, G. J. Tang, H. C. Jiang, and X. H. Yuan, "Analysis and simulation on the effect of rotor interturn short circuit on magnetic flux density of turbo-generator," Journal of Electrical Engineering-Elektrotechnicky Casopis, vol. 67, no. 5, pp. 323-333, 2016.

[3] W. Yucai and L. Yonggang, "Experimental study of rotor interturn short circuit fault diagnosis in turbine generator based on characteristic frequency of end-leakage-flux," Transactions of China Electro-Technical Society, vol. 11, pp. 107-115, 2014.

[4] S. Yuguang, Y. Xiwen, W. Kun, Z. Huang, and X. Wang, "A new type of search coil for detecting inter-turn faults in synchronous machines," Proceedings of the CSEE, vol. 6, pp. 917-924, 2014.

[5] L. Yang, H. Liangliang, S. Yuguang et al., "Characteristic analysis of unbalanced magnetic pull caused by non-salient pole synchronous generator rotor inter-turn short circuit fault," Automation of Electric Power System, vol. 40, no. 3, pp. 8189, 2017.

[6] W. Yucai and L. Yonggang, "Diagnosis of rotor winding interturn short-circuit in turbine generators using virtual power," IEEE Transactions on Energy Conversion, vol. 30, no. 1, pp. 183-188, 2015.

[7] Z. Hongsen, Research on Faults Features and Electromagnetic Performance Related to Stator Internal Short-Circuit in Nuclear Power Turbo-Generator, Harerbin University of Science and Technology, Haerbin, China, 2018.

[8] Y.-g. Li, Y.-j. Zhao, L. Chen, and X. Ji, "Fault Diagnosis of Rotor Winding Inter-Turn Short Circuit in TurbineGenerator Based on BP Neural Network," in 2008 Interna- tional Conference on Electrical Machines and Systems, pp. 783-787, Wuhan, 2008.

[9] W. Yucai, M. Qianqian, and C. Bochong, "Fault diagnosis of rotor winding inter-turn short circuit for sensorless synchronous generator through screw," IET Electric Power Applications, vol. 11, no. 8, pp. 1475-1482, 2017.

[10] W. Shuting, L. Yonggang, L. Heming, and T. Guiji, “The New Diagnosis Method of Rotor Winding Inter-Turn Short Circuit Fault and Imbalance Fault Based on Stator and Rotor Vibration Characteristics," in 2005 International Conference on Electrical Machines and Systems, pp. 2207-2210, Nanjing, China, 2005.

[11] W. Shuting, L. Yonggang, L. Heming, and T. Guiji, “A compositive diagnosis method on turbine-generator rotor winding inter-turn short circuit fault," in 2006 IEEE International Symposium on Industrial Electronics, pp. 1662-1666, Montreal, Que., 2006.

[12] Q. Meng and Y. He, "Mechanical Response before and after Rotor Inter-Turn Short-Circuit Fault on Stator Windings in Synchronous Generator," in 2018 IEEE Student Conference on Electric Machines and Systems, pp. 1-7, HuZhou, China, 2018.

[13] G. Tang, H. Jiang, Y. He, and Q. F. Meng, "Electromagnetic forces and mechanical responses of stator windings before and after rotor interturn short circuit in synchronous generators," Mathematical Problems in Engineering, vol. 2020, 19 pages, 2020.

[14] H.-C. Jiang, G.-J. Tang, Y.-L. He, K. Sun, W. J. Li, and L. Cheng, "Effect of static rotor eccentricity on end winding forces and vibration wearing," International Journal of Rotating Machinery, vol. 2021, 14 pages, 2021. 